\title{
Failure to detect changes to people during a real-world interaction
}

\author{
DANIEL J. SIMONS \\ Harvard University, Cambridge, Massachusetts \\ and \\ DANIEL T. LEVIN \\ Kent State University, Kent, Ohio
}

\begin{abstract}
Recent research on change detection has documented surprising failures to detect visual changes occurring between views of a scene, suggesting the possibility that visual representations contain few details. Although these studies convincingly demonstrate change blindness for objects in still images and motion pictures, they may not adequately assess the capacity to represent objects in the real world. Here we examine and reject the possibility that change blindness in previous studies resulted from passive viewing of $2-\mathrm{D}$ displays. In one experiment, an experimenter initiated a conversation with a pedestrian, and during the interaction, he was surreptitiously replaced by a different experimenter. Only half of the pedestrians detected the change. Furthermore, successful detection depended on social group membership; pedestrians from the same social group as the experimenters detected the change but those from a different social group did not. A second experiment further examined the importance of this effect of social group. Provided that the meaning of the scene is unchanged, changes to attended objects can escape detection even when they occur during a natural, real-world interaction. The discussion provides a set of guidelines and suggestions for future research on change blindness.
\end{abstract}

Despite our impression that we retain the visual details of our surroundings from one view to the next, we are surprisingly unable to detect changes to such details. Recently, experiments from a number of laboratories have shown that people fail to detect substantial changes to photographs of objects and real-world scenes when the ability to detect retinal differences is eliminated (Blackmore, Brelstaff, Nelson, \& Troscianko, 1995; Grimes, 1996; Henderson, 1997; McConkie \& Currie, 1996; O’Regan, Deubel, Clark, \& Rensink, 1997; Pashler, 1988; Phillips, 1974; Rensink, O’Regan, \& Clark, 1997; Simons, 1996; for a review see Simons \& Levin, 1997). That is, when retinally localizable information signaling a change is masked by an eye movement or a flashed blank screen, observers have difficulty detecting changes to the visual

The authors contributed equally to this report, and authorship order was determined arbitrarily. Thanks to Leon Rozenblit, Carter Smith, Julia Noland, and Joy Beck for helping to carry out the experiments and to Linda Hermer for reading an earlier draft of the manuscript. D.J.S. was supported by NSF and Jacob K. Javits fellowships, and parts of this research appeared in his doctoral thesis. Correspondence should be addressed to D. J. Simons, Department of Psychology, Harvard University, 820 William James Hall, 33 Kirkland St., Cambridge, MA 02138 (email: dsimons@wjh.harvard.edu) or D. T. Levin, Department of Psychology, Kent State University, P.O. Box 5190, Kent, OH 44242-0001 (e-mail: dlevin@kent.edu). details of a scene. These findings of "change blindness" suggest that observers lack a precise visual representation of their world from one view to the next. Although we have known for some time that memory for scenes is often distorted, sometimes quite sparse, subject to suggestions, and influenced by expectations and goals (Bartlett, 1932/1977; Brewer \& Treyens, 1981; Loftus, 1979; Nickerson \& Adams, 1979), studies of change blindness suggest that such details may not be retained even from one instant to the next, a claim that is consistent with earlier studies of the integration of information from successive fixations (Bridgeman \& Mayer, 1983; Dennett, 1991; Hochberg, 1986; Irwin, 1991; McConkie \& Currie, 1996; Pashler, 1988; Rayner \& Pollatsek, 1992).

Given the richness of our visual world, it is perhaps unsurprising that we cannot represent all the visual details of every object and instead must focus on a few important objects. Recent models of attention have argued that observers can fully represent the details of only a few centrally attended objects in a scene. For example, models based on object files (e.g., Treisman, 1993) suggest that we can simultaneously represent several distinct objects in our environment, updating our representations for changes in their properties and features. Such models suggest the possibility that representations of centrally attended objects are relatively detailed even if those for peripheral objects are not. 
A recent series of studies directly examined the role of attention in the detection of changes to natural images (Rensink et al., 1997). In their "flicker paradigm," an original version and a modified version of an image were presented in rapid alternation ( $240 \mathrm{msec}$ each), with a blank screen ( 80 -msec duration) interposed between them, producing a flickering appearance. On each trial, subjects were asked to identify the changing part of the image as soon as they saw it. Consistent with earlier studies of integration across views (for a review, see Irwin, 1991), observers rarely noticed changes during the first cycle of alternation and often required many cycles to detect the change. The change detection process requires observers to shift their attention among the objects in the scene, actively searching for a change. As predicted by models of object files, changes to objects that independent raters consider to be the center of interest of a scene are detected in significantly fewer alternations than changes to peripheral objects. That is, changes to the details of attended objects are detected more readily.

Clearly, focused attention to an object is helpful and possibly necessary for change detection, as evidenced by such "center of interest" effects (O'Regan, Rensink, \& Clark, 1996; Rensink et al., 1997; Tarr \& Aginsky, 1996, July) and by findings of more successful change detection when explicit cues specify the location or the type of change (Aginsky, Tarr, \& Rensink, 1997). However, attention may not be sufficient for change detection. In fact, observers often fail to detect changes even when attention is focused directly on the changing object (Levin \& Simons, 1997; O'Regan et al., 1997; Simons, 1996). In a recent series of studies, we used motion pictures to directly examine the ability to detect changes to attended objects (Levin \& Simons, 1997). These brief motion pictures depicted a simple action performed by a "single" actor. During the film, the actor was replaced by a different person. For example, in one film an actor walked through an empty classroom and began to sit in a chair. The camera then changed, or "cut," to a closer view and a different actor completed the action. Even though the actors were easily discriminable and were the focus of attention, only $33 \%$ of the 40 participants reported noticing the change from one actor to another (Levin \& Simons, 1997).

Although the motion picture experiments demonstrate that attention alone is not sufficient for a complete representation of the visual details of an object, they do not fully assess our ability to represent objects in the real world. Motion picture perception is similar in many ways to perception in the real world, but motion pictures are still a subset of a complete visual experience (Arnheim, 1933/1966). Most importantly, they are viewed passively and may not completely engage the processes necessary for a complete representation of attended objects. Furthermore, cuts from one view to another in motion pictures may artificially hamper our ability to detect changes. Although cuts are similar in some ways to eye movements, they also instantaneously change the simulated observation point. This artificial jump in viewing position may somehow disrupt the ability to detect changes even if it has little effect on our understanding of a scene. Similar objections might be raised about most studies documenting change blindness (for a discussion, see Simons \& Levin, 1997). In all previous studies of change blindness, exposure to scenes has been mediated via photographs, computer displays, or television monitors. Perhaps people can more fully represent the details of a scene when they are direct participants, interacting with the objects in the real world.

Here we assess this possibility by taking the study of change blindness into the real world. Rather than changing the sole actor in a video, we changed the subjects' conversation partner during a typical daily interaction.

\section{EXPERIMENT 1}

In Experiment 1, we created a situation that allowed us to surreptitiously substitute one individual for another in the middle of a natural, real-world interaction. The situation we chose was asking directions of a pedestrian on a college campus. ${ }^{1}$ We temporarily interrupted this interaction by carrying a door between the experimenter and the pedestrian. While the experimenter was occluded by the door, another experimenter took his place and continued the interaction after the door had passed. If changedetection failures are based on the passive nature of mediated stimuli, these substitutions should be clearly detectable.

\section{Method}

Subjects. A total of 15 pedestrians were approached on the campus of Cornell University. They ranged in approximate age from 20 to 65 . Only pedestrians walking alone or together with one other person (two cases) were approached.

Procedure. An experimenter carrying a campus map asked unsuspecting pedestrians for directions to a nearby building (see Figure la). Pedestrians had a clear view of the experimenter starting from a distance of approximately $20 \mathrm{~m}$ as they walked down a sidewalk. After the experimenter and pedestrian had been talking for $10-15 \mathrm{sec}$, two other experimenters carrying a door rudely passed between them. As the door passed, the first experimenter grabbed the back of the door, and the experimenter who had been carrying that part of the door stayed behind and continued to ask for directions (Figure 1c). The first experimenter kept his map during the interruption, and the second experimenter produced an identical copy of the map after the door passed. The door blocked the pedestrian's view for approximately $1 \mathrm{sec}$ (Figure 1b). From the subject's perspective, the door briefly occluded his/her conversation partner, and when it was gone, a different person was revealed. As the door passed, subjects typically made eye contact with the second experimenter before continuing to give directions. ${ }^{2}$ The entire interaction took 2-5 min. The two experimenters wore different clothing and differed in height by approximately $5 \mathrm{~cm}$ (Figure 1d). Their voices were also clearly distinguishable.

After a pedestrian finished giving directions, the experimenter told him/her, "We're doing a study as part of the psychology department [experimenter points to the psychology building next door] of the sorts of things people pay attention to in the real world. Did you notice anything unusual at all when that door passed by minute ago?" Responses were 
a

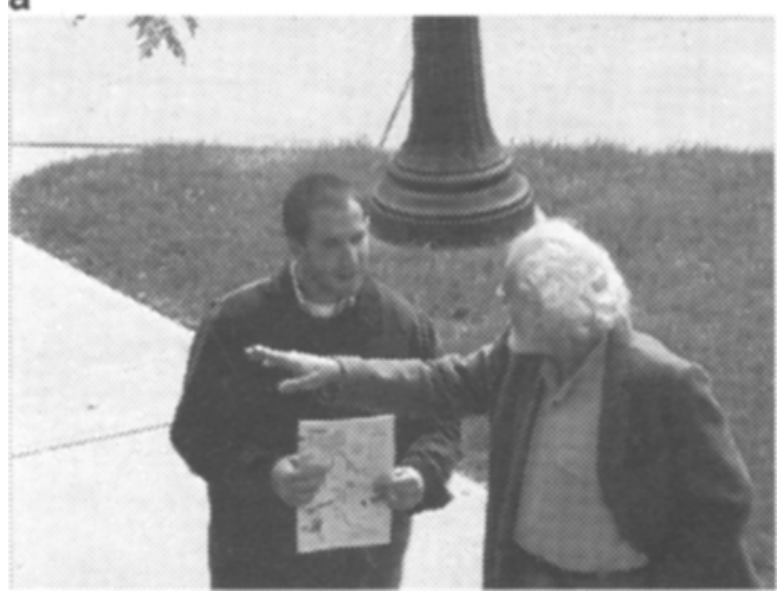

C

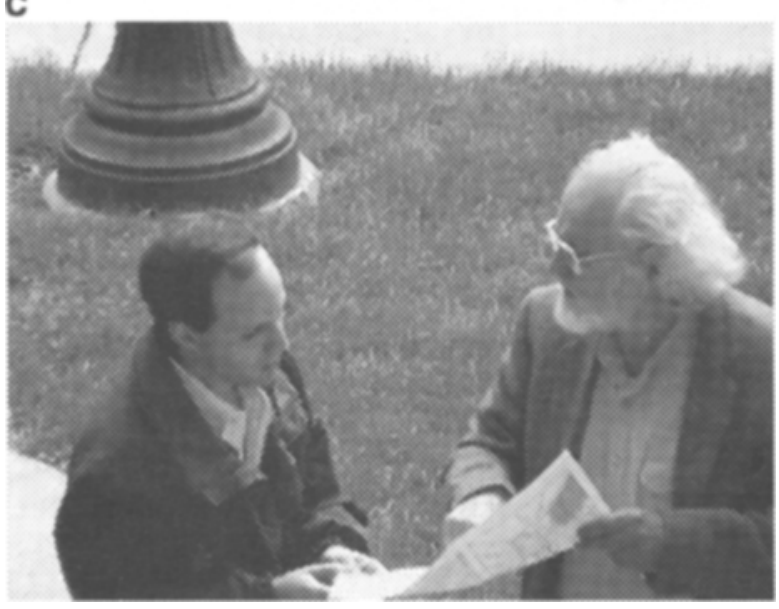

b

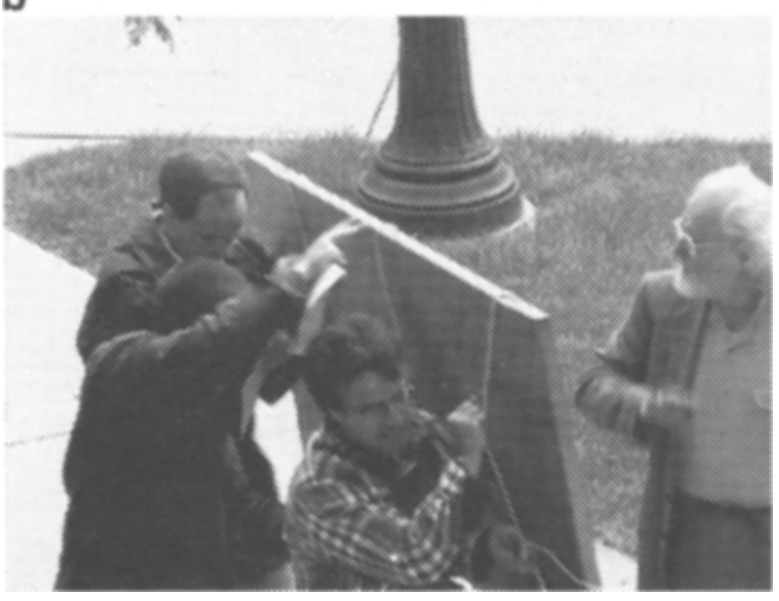

d

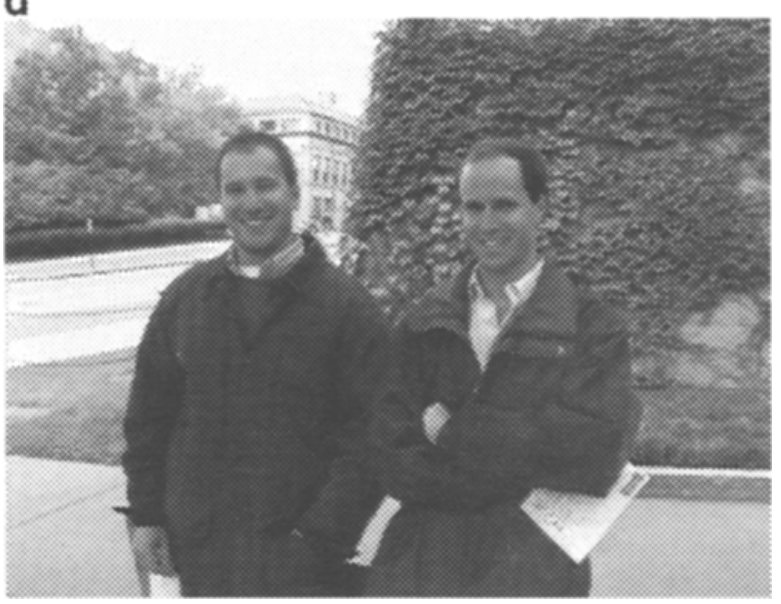

Figure 1. Frames from a video of a subject from Experiment 1. Frames a-c show the sequence of the switch. Frame d shows the two experimenters side by side.

noted by the experimenter, and if subjects failed to report the change, they were directly asked, "Did you notice that I'm not the same person who approached you to ask for directions?" After answering this question, all subjects were informed about the purpose of the experiment.

\section{Results and Discussion}

If change blindness results from the passive nature of mediated stimuli, then these real-world substitutions should be detected. When asked if they had noticed anything unusual, most pedestrians reported that the people carrying the door were rude. Yet, despite clear differences in clothing, appearance, and voice, only 7 of the 15 pedestrians reported noticing the change of experimenters. Those who did not notice the change continued the conversation as if nothing had happened (in fact, some pedestrians who did notice the change also continued the conversation!). Pedestrians who did not notice the change were quite surprised to learn that the person standing in front of them was different from the one who initiated the conversation. One pedestrian who reported noticing nothing unusual nonetheless claimed to have noticed the change when asked directly.

Interestingly, those who noticed the change were all students of roughly the same age as the experimenters (approximately 20-30 years old). Those who failed to detect the change were slightly older than the experimenters (approximately 35-65 years old). One possible explanation for this difference is that younger pedestrians were more likely to expend effort encoding those features that would differentiate the experimenters because the experimenters were roughly of their own generation. In contrast, older pedestrians would likely encode the experimenters without focusing on features that could differentiate the two of them, instead viewing them as members of a social group other than their own. This hypothesis draws on findings from social psychology that members of one's own social group ("in-group") are treated differently from members of social groups distinctly apart from one's own ("out-group"). Upon encountering a member of an in-group, people tend to focus at- 


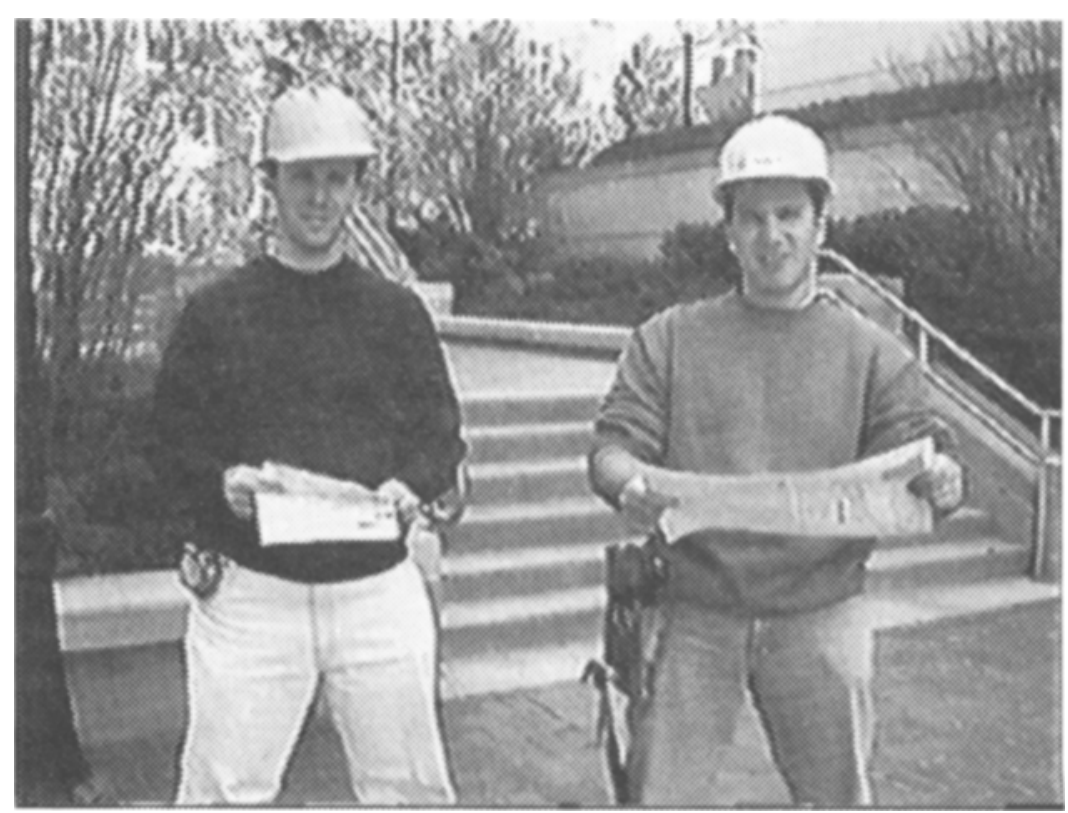

Figure 2. The experimenters dressed as construction workers for Experiment 2.

tention on individuating features and to pay little attention to the person's social-group membership. In contrast, for members of out-groups, people direct more attention to attributes associated with the out-group as a whole and generally do not focus on features that distinguish one individual from others in the group (see, e.g., Rothbart \& John, 1985). These differences in processing of members of in-groups and out-groups extend to many aspects of cognition. For example, people are likely to assume that members of out-groups are collectively less variable on a variety of traits and variables (Judd \& Park, 1988; Linville, Fischer, \& Salovey, 1989). This tendency to code groupspecifying information for members of out-groups can even determine what represents a visual feature for a particular category (Levin, 1996).

Applying these differences in the coding of in-groups and out-groups to the findings of Experiment 1, we hypothesize that the younger subjects considered themselves members of the same social group as the experimenters and older subjects considered the experimenters to be members of an out-group. To test this hypothesis, we changed the appearance of the experimenters so that they could be classified as members of an out-group by the younger subjects.

\section{EXPERIMENT 2}

To examine the role of social group membership in the detection of changes, a second experiment was conducted using the same procedure as the first, but with one critical change: The same two experimenters dressed as construction workers (see Figure 2). The experimenters again wore different clothing: One wore a construction hat with writing on the front, a large tool belt, and a light blue shirt, and the other wore a newer hat without writing, no tool belt, and a black shirt. The experiment was conducted in the same location as Experiment 1, which happened to be approximately $50 \mathrm{~m}$ from a construction site. As in Experiment 1 , an experimenter approached a pedestrian to ask for directions to a building on campus. During the conversation, the experimenters were switched. Unlike in the first experiment, all 12 pedestrians who participated in Experiment 2 were from the younger age group (Cornell graduate or undergraduate students), the group that had always detected the change in Experiment 1. The questions asked of the subjects were identical to those of Experiment 1 except that subjects were informed immediately after providing directions that the experimenters were not actually construction workers but were doing a study as part of the psychology department.

\section{Results and Discussion}

In contrast to the younger pedestrians in Experiment 1, all of whom noticed the change, only 4 of the 12 pedestrians in Experiment 2 reported noticing the switch when asked if they had seen anything unusual. Five subjects failed to report the change and were surprised to learn of the switch. An additional 3 subjects reported noticing nothing unusual but then claimed to have noticed the switch of experimenters. Unlike pedestrians who clearly noticed the change, these 3 pedestrians could not accurately describe any of the differences between the experimenters, suggesting that the demands of the task led them to report noticing the change even though they prob- 
ably had not. Thus, subjects from the same age group that had successfully detected the change in Experiment 1 detected it only 33\% of the time in Experiment 2 .

When the experimenters appeared to be members of an out-group, thereby decreasing the likelihood that students would code individuating features, the ability to detect a change to the centrally attended object in a scene was dramatically reduced. One subject who failed to detect the change essentially stated our predicted hypothesis: She said that she had just seen a construction worker and had not coded the properties of the individual. That is, she quickly categorized the experimenter as a construction worker and did not retain those features that would allow individuation. Even though the experimenter was the center of attention, she did not code the visual details and compare them across views. Instead, she formed a representation of the category, trading the visual details of the scene for a more abstract understanding of its gist or meaning.

\section{GENERAL DISCUSSION}

These simple experiments build on classic findings of failures of eyewitness identification (e.g., Loftus, 1979) and distortions in memory (Bartlett, 1932/1977) as well as recent demonstrations of change blindness for objects (Pashler, 1988; Phillips, 1974; Simons, 1996), photographs (Aginsky et al., 1997; Grimes, 1996; O’Regan et al., 1996 Rensink et al., 1997), and motion pictures (Levin \& Simons, 1997; Simons, 1996; Simons \& Levin, 1997). Yet, unlike earlier demonstrations, this experiment shows that people may not notice changes to the central object in a scene even when the change is almost instantaneous and happens in the middle of an ongoing, natural event. Attention alone does not suffice for change detection, even in the real world. Instead, successful change detection probably requires effortful encoding of precisely those features or properties that will distinguish the original from the changed object.

One potential objection to our results derives from the pragmatics of the interaction. Specifically, subjects may have detected the change but the social demands of the situation precluded them from reporting it This possibility is substantially diminished by the subjects in each experiment who reported noticing nothing unusual but then reported noticing the switch. Although these subjects probably did not notice the change, the social demands of the situation encouraged them to report having noticed the switch when asked directly. Thus, the demands of the situation seem biased to increase reports of the switch rather than to decrease them

Another possible objection is that the task of giving directions distracted subjects from focusing their attention on the experimenters. That is, subjects were focused on the map rather than their conversational partner. Anecdotally at least, subjects appeared focused on the interaction and the conversation, often making eye contact with the experimenters, hearing their voices, and taking turns in a conversation. Although we believe the results are not specific to this situation, ongoing experiments using a different type of interaction are directly examining the possible distraction caused by the map and possible disruptions to the representation of the first experimenter caused by the unusual nature of the interruption.

A more fundamental question involves assessing the similarity of the experimenters. Clearly, no one would be surprised if pedestrians failed to notice a substitution of identically dressed identical twins. The inability to notice small changes is unsurprising because such changes naturally occur between views. For example, people rarely notice variation in the position and orientation of moveable objects such as body parts (Levin \& Simons, 1997). If we constantly noticed such changes, they would likely detract from our ability to focus on other, more important aspects of our visual world. Change detection as a method relies on the tendency of our visual system to assume an unchanging world. The fact that we do not expect one person to be replaced by another during an interaction may contribute to our inability to detect such changes. A critical question for future research is why some changes are more likely to be detected than others. Clearly we would be quite surprised if subjects missed a switch between enormously different people (e.g., a switch from a $4 \mathrm{ft} 9 \mathrm{in}$. female of one race to a $6 \mathrm{ft} 5 \mathrm{in}$. male of another). The change in this case would alter not only the visual details of the person, but also their category membership. If, as suggested by other recent findings of change blindness, we retain only abstracted information and not visual details from one view to the next, changes to category membership may well be detectable. Abstraction of category information is clearly central to coding other people (e.g., the effects of in-group and out-group discussed earlier) and may underlie the representation of other objects across views as well.

What, then, separates inconsequential changes to details from changes that are worth noting? Although there is no easy answer to this question, we would like to propose several guidelines or heuristics for identifying consequential changes for future studies of change blindness. These guidelines, used individually or together, can help constrain the generation of significant changes to scenes.

First, significant changes to a scene should be easily verbalizable, and often verbalized (see Simons, 1996). Changes that are easily verbalized likely cross a category boundary, making them more likely to be detected. The best example of this principle is the change in the color of the experimenter's shirt in Experiment 2. Both shirt colors (blue and black) have basic color names.

Second, the original and changed objects should be easily discriminable in simultaneous viewing. Everyone is familiar with the comicspage game of finding differences between two extremely similar images. In such cases, the change is camouflaged, making it difficult to detect even when both the original and changed version are present. In our experiment, as in most studies of change blindness (see Simons \& Levin, 1997), changes generally meet this criterion (e.g., the difference in shirt colors is plainly visible in Figure 2).

Third, changes should affect the immediate functional needs of the perceiver. For example, changes to the spatial configuration of objects or their parts can be significant, even if they are not easy to verbalize. Spatial layout information is crucial to navigation and other immediate needs of the organism. For our experiments, variation in the configuration of facial features is precisely the information used in identifying other people; hence the person change should be readily detectable.

Fourth, naive subjects should predict successful change detection. If change blindness is counterintuitive, we can be certain that the change is not trivial. For our experiments, individuals unfamiliar with our research consistently predicted that the change of experimenters would be plainly detectable. To examine this possibility for our experiments, we informally polled a class of 50 introductory psychology students by reading them the following description of our event: "You are walking on the Cornell campus and a man with a puzzled look asks you to help him find Olin library. You stop and give him directions. While you are giving directions, two people carrying a door rudely walk between you and the lost pedestrian. After the door has passed, the person you were giving directions to is now a different person wearing different clothes." By a show of hands, they claimed without exception that they would detect the change.

By applying these four heuristics, researchers can be fairly certain that a change is detectable and that change blindness would be an important finding. In our experiments, the change from one experimenter to another met all of these criteria. Yet, a substantial number of pedestrians failed to detect the switch. Taken together, these experiments show that even substantial changes to the objects with which we are directly interacting will often go unnoticed. Our visual system does not automatically compare the features of a visual scene from one instant to the next in order to form a continuous representation; we do not form a detailed visual representation of our world. Instead, our abstract ex- 
pectations about a situation allow us to focus on a small subset of the available information that we can use to check for consistency from one instant to the next.

\section{REFERENCES}

AgINSKy, V., TARR, M. J., \& RenSink, R. A. (1997). The stability of color, location, and object presence in mental representations of natural scenes. Investigative Ophthalmology \& Visual Science, $\mathbf{3 8}$ S1009.

Arnheim, R. (1966). Film as art. Berkeley: University of California Press. (Original work published 1933)

BARTLETT, F. C. (1977). Remembering: A study in experimental and social psychology. Cambridge: Cambridge University Press. (Original work published 1932)

Blackmore, S. J., Brelstaff, G., Nelson, K., \& Troscianko, T. (1995). Is the richness of our visual world an illusion? Transsaccadic memory for complex scenes. Perception, 24, 1075-1081.

BREWER, W. F., \& TREYENS, J. C. (1981). Role of schemata in memory for places. Cognitive Psychology, 13, 207-230.

Bridgeman, B., \& MAYER, M. (1983). Failure to integrate visual information from successive fixations. Bulletin of the Psychonomic Society, 21, 285-286.

DenNETT, D. C. (1991). Consciousness explained. Boston: Little, Brown.

Grimes, J. ( 1996). On the failure to detect changes in scenes across saccades. In K. Akins (Ed.), Perception: Vol. 2. Vancouver studies in cognitive science (pp. 89-110). New York: Oxford University Press.

HENDERSON, J. M. (1997). Transsaccadic memory and integration during real-world object perception. Psychological Science, 8, 51-55.

HOCHBERG, J. (1986). Representation of motion and space in video and cinematic displays. In K. R. Boff, L. Kaufman, \& J. P. Thomas (Eds.), Handbook of perception and human performance: Vol. 1. Sensory processes and perception (pp. 22.21-22.64). New York: Wiley.

IRWIN, D. E. (1991). Information integration across saccadic eye movements. Cognitive Psychology, 23, 420-456.

JUDD, C. M., \& PARK, B. (1988). Out-group homogeneity: Judgments of variability at the individual and group levels. Journal of Personality \& Social Psychology, 54, 778-788.

LEvin, D. T. (1996). Classifying faces by race: The structure of face categories. Journal of Experimental Psychology: Leaning, Memory, \& Cognition, 22, 1364-1382.

LEVIN, D. T., \& Simons, D. J. (1997). Failure to detect changes to attended objects in motion pictures. Psychonomic Bulletin \& Review, 4, 501-506.

Linville, P. W., Fischer, G. W., \& Salovey, P. (1989). Perceived distributions of the characteristics of in-group and out-group members: Empirical evidence and a computer simulation. Journal of Personality \& Social Psychology, 57, 165-188.

LofTus, E. F. (1979). Eyewitness testimony. Cambridge, MA: Harvard University Press.

McConkie, G. W., \& Currie, C. B. (1996). Visual stability across sac- cades while viewing complex pictures. Journal of Experimental Psychology: Human Perception \& Performance, 22, 563-581.

NiCKERSON, R. S., \& ADAMS, M. J. (1979). Long-term memory for a common object. Cognitive Psychology, 11, 287-307.

O'Regan, J. K., Deubel, H., Clark, J. J., \& Rensink, R. A. (1997). Picture changes during blinks: Not seeing where you look and seeing where you don't look. Investigative Ophthalmology \& Visual Science, 38, 5707 .

O'Regan, J. K., Rensink, R. A., \& Clark, J. J. (1996). "Mud splashes" render picture changes invisible. Investigative Ophthalmology \& Visual Science, 37, S213.

PAsHLER, H. (1988). Familiarity and visual change detection. Perception \& Psychophysics, 44, 369-378.

Phillips, W. A. (1974), On the distinction between sensory storage and short-term visual memory. Perception \& Psychophysics, 16, 283-290.

Rayner, K., \& Pollatsek, A. (1992). Eye movements and scene perception. Canadian Journal of Psychology, 46, 342-376.

RENSINK, R. A., O'Regan, J. K., \& CLARK, J. J. (1997). To see or not to see: The need for attention to perceive changes in scenes. Psychological Science, 8, 368-373.

RothraRT, M., \& JoHN, O. P. (1985). Social categorization and behavioral episodes: A cognitive analysis of the effects of intergroup contact. Journal of Social Issues, 41, 81-104.

Simons, D. J. (1996). In sight, out of mind: When object representations fail. Psychological Science, 7, 301-305.

Simons, D. J., \& Levin, D. T. (1997). Change blindness. Trends in Cognitive Sciences, 1, 261-267.

TARR, M. J., \& AGINSKY, V. (1996, July). From objects to scenes: Speculations on similarities and differences. Paper presented at the Scene Recognition Workshop, Max-Planck-Institut für Biologische Kybernetik, Tübingen.

Treisman, A. (1993). The perception of features and objects. In A. Baddeley \& L. Weiskrantz (Eds.), Attention: Selection, awareness, and control: A tribute to Donald Broadbent (pp. 5-35). Oxford: Oxford University Press, Clarendon Press.

\section{NOTES}

1. The idea for this experimental situation came from a comedy television show that used a similar event. Thanks to Ron Rensink for bringing it to our attention.

2. Although some subjects made more eye contact than others, the vantage point of our hidden camera precluded a precise analysis of the effect of eye contact on detection of the change; the initial eye contact between the second experimenter and the pedestrian was masked by the door. In all cases, subjects made extensive eye contact after completing their directions, and most pedestrians did make eye contact immediately before and after the arrival of the door, suggesting that eye contact does not guarantee successful detection of the change.

(Manuscript received October 1, 1997; revision accepted for publication January 8,1998 .) 